\title{
Effects of quality changes in rental housing markets with indivisibilities
}

\author{
Tamon Ito* \\ Institute of Policy and Planning Sciences, University of Tsukuba, Ibaraki \\ 305-8573, Japan
}

Received 21 February 2005; revised 30 November 2006

\begin{abstract}
This paper presents comparative statics results on a rental housing market model with a finite number of apartments. The apartments are classified into categories based on their housing attributes. The apartments in a given category are treated as equals having the same rent. The main result is that when an improvement occurs in one category, the rent of the category increases, whereas rents of better categories decrease. We compare this result with another comparative statics result, and also with Braid's results in his model with a continuum of housing qualities.
\end{abstract}

Key words: Rental housing market; Comparative statics; Rent equation;

Differential rent; Quality improvement

JEL classification: R31; R30; D58

* Tel./fax: +81-29-853-5580.

Email address: taito@shako.sk.tsukuba.ac.jp (Tamon Ito).

Preprint submitted to Elsevier

30 November 2006 


\section{Introduction}

In this paper, we consider comparative statics in the rental housing market model of Kaneko-Ito-Osawa (2006), where apartments are treated as indivisible goods and are exchanged with one composite good. In particular, we consider the comparative statics of apartment rents when the quality of a certain apartment class is improved. This comparative statics will be contrasted with another result. These comparative statics give similar effects on the rent of the apartments in question but opposite effects on the rents of the other apartments.

First, we explain the background of the model as well as the relationship to the relevant literature, since the approach we take seems not to be well-known in the urban economics literature. Then, we give diagrammatic explanations of the two above-mentioned comparative statics results. Finally, we will mention some possible extensions of our results.

Our approach is regarded as belonging to the literature of "assignment markets" from Böhm-Bawerk (1921), Neumann-Morgenstern (1944) and ShapleyShubik (1972). In this literature, markets with one indivisible good and one divisible good have been studied, while allowing product differentiation in their indivisible units. However, the literature reflects almost no interactions with urban economics, except for Kaneko (1983) and Gerber (1985). Recently, Kaneko et al. (2006) gave a more systematic application of the approach to urban rental housing markets, and also studied various properties arising from comparative statics. The present paper is a further study in this line of research. 
We also find that our approach is related to the bid rent approach in the urban economics literature originated with Alonso (1964), Muth (1969) and Mills (1972). ${ }^{1}$ Those models are typically described by continuous variables and continuous functions. On the other hand, the main feature of our model is discreteness, i.e., each housing unit is indivisible and the number of housing units is finite. An important difference in methodology is that calculus is a main method in the standard urban economic literature, while ours is truly combinatorial.

Here, we give a brief description of our housing market model and our comparative statics. The details will be given in Sections 2,3 and 4 . In our model, the apartments are classified and ranked qualitatively. Each household is assumed to have the identical utility function expressed as $h_{k}+g(c)$. This utility function is separable as the sum of the utility level $h_{k}$ from the $k$-th ranked apartment itself and the utility level $g(c)$ from the $c$ of composite good. The first term, $h_{k}$, reflects the "utility" from the housing attributes of the $k$-th apartment, such as size, commuting time to the central business district (CBD) and age. We interpret $h_{k}$ as representing the quality of the $k$-th apartment. ${ }^{2}$ Here, we can assume without loss of generality ${ }^{3}$ that the qualities are arranged in numerical order: $h_{1}>h_{2}>\cdots>h_{T}$. Thus, the lower the rank, the higher the quality of the apartment.

In the rental housing market described above, we consider the competitive

$\overline{1}$ For recent developments and treatments, see Arnott (1987) and Fujita (1989).

2 This definition of "quality" is independent of an income. See Example in Section

2.

3 This can be obtained by renaming the apartments under the assumption that no indifferences are allowed. 
rents $r_{1}, \ldots, r_{T}$ of apartments $1, \ldots, T$, which are described in the first row of Fig. 1. Now, we suppose that the quality ("utility") $h_{k}$ is improved to $\hat{h}_{k}$, but the other $h_{t}$ 's remain unchanged. The new competitive rents $\hat{r}_{1}, \ldots, \hat{r}_{T}$ described in the second row of the figure differ from the original competitive rents $r_{1}, \ldots, r_{T}$. In the shaded area of Fig. $1, r_{k}$ increases to $\hat{r}_{k}$, but the rent $r_{t}$ decreases to $\hat{r}_{t}$ for $t<k$. It is, more or less, an assumption that the rent $r_{t}$ is constant for $t>k$. This result will be given in Section 4.2 and will be proved in Section 6.

The above result will be compared with another comparative statics result, which is a variant of an earlier result in Kaneko et al. (2006). In this comparative statics, all the $h_{t}$ 's remain unchanged, but only the income of the household choosing the $k$-th apartment increases. Then, the changes in the rents $r_{1}, \ldots, r_{T}$ are expressed as shown in Fig. 2 .

Compare the shaded area in Fig. 2 with that in Fig. 1. The rents of the better apartments than $k$ change in the opposite directions, while we find the same behavior of rent in the $k$-th. This contrast will be explained in Section 4.3.

In the above comparative statics of Fig. 1, only one apartment is improved. However, this result can be extended to the case where several apartments are improved, which will be discussed in Section 5. This extension is needed, for example, when the commuting time to the CBD is shortened.

In the standard urban economics literature, we find some works such as Sweeney (1974), Braid (1981) and van Lierop (1982) which discuss comparative statics similar to ours. In particular, certain results in Braid (1981) are closely related to our comparative statics results, which will be discussed at the end of Section 4 . 
The format of this paper is as follows. Section 2 formulates our rental housing market model. Section 3 introduces the rent equation, which is our main analytical tool. Section 4 gives our results, expressed by Fig. 1 and Fig. 2, and compares them with Braid's results. Section 5 discusses the extension of our result. Section 6 proves the above two results. Section 7 concludes the paper.

\section{The market model}

We follow a simplified version of the rental housing market model $(M, N)$ of Kaneko et al. (2006). The symbols $M$ and $N$ denote the set of households, $M=\{1, \ldots, m\}$, and the set of landlords, $N=\left\{1^{\prime}, \ldots, n^{\prime}\right\}$. Each household $i \in M$ having income $I_{i}>0$ will rent at most one unit of an apartment. Without loss of generality, we can assume that the households are ordered in their incomes as $I_{1} \geq I_{2} \geq \cdots \geq I_{m}$. This is simply a renaming of the households.

It is assumed that apartments are already built and fixed. The apartments are indivisible goods and are traded for one perfectly divisible composite good (money). The apartments are classified into categories $1, \ldots, T$ by their housing attributes, e.g., their size and commuting times to the CBD. Thus, we consider $T$ kinds of apartments, and each kind is a category consisting of various units. It is assumed that each landlord $j \in N$ owns some units only in one category and places them on the market.

With a slight stretching of these terms, we may write the $k$-th category of apartments as the " $k$-th apartment." Also, we may write "unit" to express an individual apartment. 
Consumption other than housing is summarized as one composite good. The consumption set for each household is given as $\Omega:=\left\{\boldsymbol{e}^{0}, \boldsymbol{e}^{1}, \cdots, \boldsymbol{e}^{T}\right\} \times \boldsymbol{R}_{+}$, where $\boldsymbol{R}_{+}$is the set of nonnegative real numbers, $e^{k}$ is the $T$ dimensional unit vector with its $k$-th element equal 1 for $k=1, \ldots, T$, and $e^{0}$ is a zero vector 0. A generic element $\left(\boldsymbol{e}^{k}, c\right)=\left(\boldsymbol{e}^{k}, I_{i}-p_{k}\right)$ with $k \geq 1$ means that household $i$ rents one unit in the $k$-th category and consumes $c=I_{i}-p_{k}$ amount of the composite good, where $p_{k}$ is the rent of $\boldsymbol{e}^{k}$. The zero vector $\boldsymbol{e}^{0}$ means that the household does not rent any apartment in $(M, N) .{ }^{4}$

In the following, we define the utility function and the cost function specified for our analysis.

Assumption A (Utility Function). Every household has an identical utility function $u: \Omega \rightarrow \boldsymbol{R}$ expressed as

$$
u\left(e^{k}, c\right)=h_{k}+g(c)
$$

where $h_{1}>h_{2}>\cdots>h_{T}$, and $g: \boldsymbol{R}_{+} \rightarrow \boldsymbol{R}$ is a strictly concave, continuous, and monotone increasing function of $c$ with $\lim _{c \rightarrow+\infty} g(c)=+\infty$, and $h_{0}+g\left(I_{m}\right)>$ $h_{1}+g(0)$.

The additional requirements stated after (1) are as follows: 1) The apartment utility levels $h_{1}, \cdots, h_{T}$ are ranked strictly in the numerical order; 2$)$ the last inequality is a boundary condition, where $h_{0}$ is the utility level of no apartment, $e^{0}$. This inequality states that leaving the market is preferred to renting an apartment with no consumption. ${ }^{5}$

$\overline{4}$ In our equilibrium analysis, this option $\mathbf{0}$ is not chosen in effect.

5 Since $h_{1}>\cdots>h_{T}$ and $I_{1} \geq \cdots \geq I_{m}$, the inequality $h_{0}+g\left(I_{m}\right)>h_{1}+g(0)$ implies that $h_{0}+g\left(I_{i}\right)>h_{k}+g(0)$ for all $i \in M$ and $k=1, \ldots, T$. 
Now, we explain three important properties of Assumption A.

Identical utility function: We assume that every household has the same utility function, i.e., $u_{i}(\cdot, \cdot)=u_{i^{\prime}}(\cdot, \cdot)$ for all $i, i^{\prime} \in M$. Therefore, for each $k=1, \ldots, T$, the utility level $h_{k}$ is independent of $i$ for all $i \in M .{ }^{6}$ This assumption may look restrictive. Nevertheless, individual households with different incomes may behave differently because of the normality property of the utility function, which will be explained below.

Separability: The entire utility function $u$ is expressed as the sum of utility level $h_{k}$ from the $k$-th apartment and utility level $g(c)$ from the consumption of composite good $c$. The utility level $h_{k}$ reflects the quality of the $k$-th apartment.

Normality: The strict concavity of function $g$ has the normality implication, which is expressed as

$$
\begin{gathered}
\text { if } h_{k}+g(c)=h_{k^{\prime}}+g\left(c^{\prime}\right), c<c^{\prime} \text { and } \delta>0, \\
\text { then } h_{k}+g(c+\delta)>h_{k^{\prime}}+g\left(c^{\prime}+\delta\right) .
\end{gathered}
$$

In (2), apartment $k$ has a better quality than $k^{\prime}$, since living in $k$ with smaller consumption $c$ is indifferent to living in $k^{\prime}$ with larger $c^{\prime}$. When an income is increased by the same magnitude $\delta>0$, the household's demand for housing shifts to a better apartment. Thus, the apartment quality is a normal good. This is the key to our comparative statics. We will give an example of a utility function satisfying Assumption A at the end of this section.

The supply side is formulated as follows. As far as competitive equilibrium is concerned, we can assume that only one landlord named $k$ supplies all the $\overline{6}$ Also, see Example at the end of this section. 
units in the $k$-th category. That is, we assume $N=\{1, \ldots, T\}$, and landlord $k$ has the cost function $C_{k} \cdot{ }^{7}$ For our analysis, we employ a simple form of the cost function:

Assumption B (Cost Function). Each landlord $k \in N$ has a cost function $C_{k}: Z_{+} \rightarrow R_{+}$expressed as

$$
C_{k}\left(y_{k}\right)=\left\{\begin{array}{c}
a_{k} y_{k} \quad \text { if } y_{k} \leq w_{k}, \\
\text { "large" } \text { if } y_{k}>w_{k},
\end{array}\right.
$$

where $\boldsymbol{Z}_{+}$is the set of nonnegative integers, $w_{k}$ is the number of all units owned by landlord $k, y_{k}$ is the number of units supplied to the market by landlord $k$, and "large" is a sufficiently large number.

The cost function (3) means that landlord $k$ supplies every unit up to $w_{k}$ with the constant marginal cost $a_{k}>0$, but never supplies more than $w_{k}$, since the marginal cost $C_{k}\left(y_{k}+1\right)-C_{k}\left(y_{k}\right)$ for $y_{k} \geq w_{k}$ is very large relative to the market rent. ${ }^{8}$

Now, we define a competitive equilibrium in $(M, N)$. Let $(p, x, y)$ be a triple of the rent vector $p=\left(p_{1}, \ldots, p_{T}\right) \in \boldsymbol{R}_{+}^{T}$, the apartment demand vector $x=$ $\left(x_{1}, \ldots, x_{m}\right)$ with $x_{i} \in\left\{\boldsymbol{e}^{0}, \boldsymbol{e}^{1}, \cdots, \boldsymbol{e}^{T}\right\}$ for all $i \in M$, and the apartment supply vector $y=\left(y_{1}, \ldots, y_{T}\right) \in Z_{+}^{T}$.

Definition 1 (Competitive Equilibrium). We say $(p, x, y)$ is a competitive equilibrium if and only if

7 See Kaneko et al. (2006) for the explanation of this cost function.

8 The marginal cost $a_{k}$ is interpreted as the reservation price or operating cost for the additional one unit. 
Utility maximization under Budget Constraint: for all $i \in M$,

1) $I_{i}-p x_{i} \geq 0$, where $p x_{i}=\sum_{k=1}^{T} p_{k} x_{i k}$.

2) $u_{i}\left(x_{i}, I_{i}-p x_{i}\right) \geq u_{i}\left(x_{i}^{\prime}, I_{i}-p x_{i}^{\prime}\right)$ for all $x_{i}^{\prime} \in\left\{\boldsymbol{e}^{0}, \boldsymbol{e}^{1}, \cdots, e^{T}\right\}$ with $I_{i}-p x_{i}^{\prime} \geq$

0 ;

Profit Maximization: for all $k=1, \ldots, T$,

$p_{k} y_{k}-C_{k}\left(y_{k}\right) \geq p_{k} y_{k}^{\prime}-C_{k}\left(y_{k}^{\prime}\right)$ for all $y_{k}^{\prime} \in Z_{+}$;

Balance of Total Demand and Supply: $\sum_{i \in M} x_{i}=\sum_{k=1}^{T} y_{k} \boldsymbol{e}^{k}$.

The existence of a competitive equilibrium in our model is guaranteed by the existence theorem given in Kaneko (1982) and Kaneko-Yamamoto (1986). However, the discreteness of our model generates indeterminacy in the competitive rent vector, which could be an obstacle for our comparative statics. But we can remove this obstacle by choosing a maximal competitive rent vector, which we will define below.

We say that $p$ is a competitive rent vector if $(p, x, y)$ is a competitive equilibrium for some allocation $(x, y)$. For the purpose of comparative statics, we choose a maximal competitive rent vector as representative of the set of all competitive rent vectors. A competitive rent vector $p$ is said to be maximal if $p \geq p^{\prime}$ for any competitive rent vector $p^{\prime}$. Also, a competitive equilibrium $(p, x, y)$ is called a maximal competitive equilibrium if $p$ is maximal. A maximal competitive rent vector is unique if it ever exists. Its existence in $(M, N)$ is discussed in Kaneko et al. (2006). ${ }^{9}$

Here, we give an example to illustrate the housing market model described above.

$\overline{9}$ The proof of this theorem is given in an earlier version of Kaneko et al. (2006), which will be sent upon request. 
Example. Let $s_{1}, s_{2}$ and $s_{3}$ be the sizes of apartments with $0<s_{1}<s_{2}<s_{3}$, and let $t_{1}, t_{2}, t_{3}$ and $t_{4}$ be the commuting times to the CBD with $0<t_{1}<$ $t_{2}<t_{3}<t_{4}$. Then the apartments are classified into $3 \times 4=12$ categories, each of which corresponds to a cell in Table 1. For example, the upper right cell is a category determined by $s_{3}$ and $t_{1}$. The apartments of size $s_{3}$ and commuting time $t_{1}$ are classified into this category.

Each cell in Table 1 has two components, $k$ and $h_{k}$, which are the quality ranking of the category and the "utility" from one unit in the $k$-th category, respectively. That is, $h_{k}$ is the first term of the utility function (1). For example, the upper right cell ranks first in quality among the 12 categories and has "utility" represented by $h_{1}$.

This is derived from a more basic utility function. We assume that the households have the common basic utility function $U$ defined over size, time and composite good. For example, we suppose that the basic utility function is given as

$$
U(s, t, c)=s-t+\sqrt{c},
$$

where $s, t$ and $c$ are variables for size, time and composite good, respectively. By calculating $s-t$ for every $(s, t)$ pair in Table 1 , we obtain the utility level $h_{k}$ from an apartment in the category $k$. In fact, the ordering $1, \ldots, 12$ is determined by the values of $h_{k}$ 's. Since the upper right cell is the category with the largest size $s_{3}$ and the shortest commuting time $t_{1}$, it provides the highest "utility" of housing. In a similar manner, we calculate $h_{2}, \ldots, h_{12}$, which give 
the ranks $h_{2}>\cdots>h_{12} \cdot{ }^{10}$ Since $h_{k}$ 's depend not only on the commuting time but also on the size, households may prefer shortening the commuting time to having a larger size, which case is found in the ranking $h_{3}=s_{3}-t_{3}>$ $h_{4}=s_{2}-t_{1}>h_{5}=s_{3}-t_{4}$ in Table 1 .

Using the numbering of the cells based on the above-mentioned ranking, we obtain $u: \Omega=\left\{\boldsymbol{e}^{0}, \boldsymbol{e}^{1}, \cdots, \boldsymbol{e}^{12}\right\} \times \boldsymbol{R}_{+} \rightarrow \boldsymbol{R}$ defined as

$$
u\left(e^{k}, c\right)=h_{k}+\sqrt{c}
$$

where $h_{k}=s-t$ for $k=1, \ldots, 12$ and $h_{0}$ is chosen so that $h_{0}+\sqrt{I_{m}}>h_{1}$.

Also, since $\sqrt{c}$ is strictly concave, this utility function $u\left(\boldsymbol{e}^{k}, c\right)=h_{k}+\sqrt{c}$ satisfies Assumption A.

\section{The rent equation}

In the housing market model $(M, N)$, a maximal competitive rent vector is obtained as a solution for a certain equation system, called the rent equation, which will play the central role for our comparative statics study. To introduce the rent equation, we present three propositions and several concepts developed by Kaneko et al. (2006).

First, the following proposition gives the basis for the rent equation.

Proposition 1 (Kaneko et al. (2006)). Let $(p, x, y)$ be any competitive equilibrium. Then,

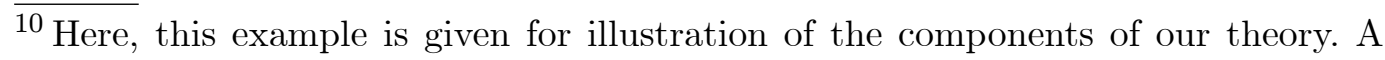
more detailed example of a treatment of the Tokyo housing market is found in Kaneko et al. (2006), Section 3.
} 
(1): If $k^{\prime}<k$ and $x_{i}=e^{k}$ for some $i$, then $p_{k^{\prime}}>p_{k}$.

(2): If $x_{i}=\boldsymbol{e}^{k}, x_{i^{\prime}}=\boldsymbol{e}^{k^{\prime}}$ and $I_{i}>I_{i^{\prime}}$, then $k \leq k^{\prime}$.

Proposition 1 states that in any competitive equilibrium, (1) a better apartment is priced higher than a worse one, and (2) a household with a higher income rents a better (or equal) apartment.

Second, we assume that for any maximal competitive equilibrium $(p, x, y)$, only the apartments in the first $f(\leq T)$ categories are traded:

Assumption C (Marginal Category). For any maximal competitive equilibrium $(p, x, y)$, there is a category $f$ such that

$$
y_{k}>0 \text { for } k=1, \ldots, f \text { and } y_{k}=0 \text { for } k=f+1, \ldots, T \text {. }
$$

We call $f$ the marginal category. It should be noted that Proposition 1(1) holds even if no units in the $k^{\prime}$-th category are rented. We assume the marginal category $f$ to eliminate such a case. This category divides the market into two segments: the categories which are active in trade, $1, \ldots, f$, and categories which are inactive, $f+1, \ldots, T$. By Assumption $\mathrm{C}$ and Proposition 1(1), we have $p_{1}>p_{2}>\cdots>p_{f}$. Note that the maximal competitive rents are uniquely determined even for the inactive categories $k>f$. The maximal rent for $k>f$ is at most the marginal cost $a_{k}$ for providing one unit in the $k$-th category, but may actually be smaller than $a_{k}$.

Finally, to characterize a maximal competitive equilibrium, we define a certain type of household. Let $(p, x, y)$ be a maximal competitive equilibrium. It follows from Proposition 1(2) that households with higher (or equal) incomes rent better (or equal) apartments in the maximal competitive equilib- 
rium. Thus, we can assume that the households rent apartments in categories $1, \ldots, f$ in the order of their incomes, $I_{1} \geq \cdots \geq I_{m}$. Under this assumption, the household that rents the last unit in each category has the lowest income among households in the category. This household plays an important role in characterizing the maximal competitive rent vector.

Formally, for each category $k=1, \ldots, f-1$, we define the last household with the lowest income in category $k$ as:

$$
G(k)=\sum_{t=1}^{k} y_{t}
$$

This is the number of households renting apartments in categories $1, \ldots, k$, and thus, $G(k)$ is the last household in category $k$. We call $G(k)$ the $k$-th boundary household. The income $I_{G(k)}$ of household $G(k)$ is called the $k$-th boundary income.

Note that there may be multiple maximal competitive allocations, though the maximal competitive rent vector is unique. The boundary household in each category depends upon the choice of a maximal competitive allocation $(x, y)$. However, this indeterminacy is inessential because of the uniqueness of the maximal competitive rent vector.

The rent equation is defined as (7) with unknowns $r_{1}, \ldots, r_{f}$ :

$$
\left.\begin{array}{c}
h_{f-1}+g\left(I_{G(f-1)}-r_{f-1}\right)=h_{f}+g\left(I_{G(f-1)}-r_{f}\right) \\
h_{f-2}+g\left(I_{G(f-2)}-r_{f-2}\right)=h_{f-1}+g\left(I_{G(f-2)}-r_{f-1}\right) \\
\vdots \\
h_{1}+g\left(I_{G(1)}-r_{1}\right)=h_{2}+g\left(I_{G(1)}-r_{2}\right) .
\end{array}\right\}
$$


The rent equation states that each boundary household $G(k)$ is indifferent between renting a unit in the $k$-th category at $r_{k}$ and renting a unit in the $(k+$ 1)-th category at $r_{k+1}$. When $r_{f}$ is given, the first equation of (7) determines $r_{f-1}\left(>r_{f}\right)$ uniquely, which is the maximal rent that the boundary household $G(f-1)$ pays for the unit in the $(f-1)$-th category. The qualitative advantage of the $(f-1)$-th over the $f$-th is reflected in the difference between $r_{f-1}$ and $r_{f}$. One may interpret the difference between $r_{f-1}$ and $r_{f}$ as the premium for the quality difference, which is described by the following equivalent form of the first equation of $(7), g\left(I_{G(f-1)}-r_{f-1}\right)-g\left(I_{G(f-1)}-r_{f}\right)=h_{f-1}-$ $h_{f}>0$. Since the utility function has income effects, households with higher incomes want a better apartment more than those with lower incomes. The other unknowns $r_{f-1}, \ldots, r_{1}$ are determined in the same manner. We call any solution, $\left(r_{1}, \ldots, r_{f-1}, r_{f}\right)$, of $(7)$ a differential rent vector. Note, again, that a differential rent vector $\left(r_{1}, \ldots, r_{f}\right)$ is uniquely determined if the rent of the marginal category $r_{f}$ is given and fixed.

The above process of determining apartment rents has a similarity to Ricardo's (1965) differential rent theory. Therefore, we use the term "differential" to describe a solution of (7).

In a more general framework, some maximal competitive equilibrium may not satisfy the rent equation. ${ }^{11}$ However, Kaneko et al. (2006) gave two sufficient conditions: either condition is sufficient for the maximal competitive rents $p_{1}, \ldots, p_{f}$ coincide with the differential rents, $r_{1}, \ldots, r_{f}$. In fact, one of their conditions is satisfied in the present framework. Thus, in our housing market $(M, N)$, we have the following proposition.

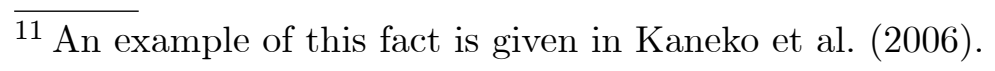


Proposition 2 (Kaneko et al. (2006)). Let $\left(\left(p_{1}, \ldots, p_{T}\right), x, y\right)$ be a maximal competitive equilibrium. Then $\left(p_{1}, \ldots, p_{f}\right)$ is a differential rent vector.

Proposition 2 implies that the differential rent vector $\left(r_{1}, \ldots, r_{f-1}, r_{f}\right)$ determined by $r_{f}=p_{f}$ coincides with the relevant part of the maximal competitive rent vector, $\left(p_{1}, \ldots, p_{f-1}, p_{f}\right)$. Here, we take $p_{f}$ as given, which is determined by the maximal competitive equilibrium. For example, suppose that there are some unoccupied units in the marginal category $f$. Then, due to competition, $r_{f}$ is determined uniquely to be the operating cost, $a_{f} .{ }^{12}$ Proposition 2 guarantees that we have the maximal competitive rents $p_{1}, \ldots, p_{f}$ as the solution of the rent equation (7) with $r_{f}=a_{f}$.

\section{Comparative statics with quality changes}

We first give an outline of comparative statics in $(M, N)$ developed by Kaneko et al. (2006). Second, we give the main result of this paper: a comparative statics result when a quality improvement of apartments occurs only in one category. Then, this result is compared with another result, which gives partially the same effect but opposite effects on the other part. Finally, we apply the above main result to a situation where the qualities of apartments are improved in multiple categories.

$\overline{12}$ A similar idea is found in Braid (1984), p.274. 


\subsection{Basic comparative statics}

In this section, we explain the basic parameter changes in comparative statics in $(M, N)$, and present one related proposition. Although these follow those of Kaneko et al. (2006), we review them for completeness. Suppose that the market $(M, N)$ changes into $(\hat{M}, \hat{N})$ with certain changes in market parameters. We make Assumptions $\mathrm{A}, \mathrm{B}$ and $\mathrm{C}$ on both $(M, N)$ and $(\hat{M}, \hat{N})$. We list the possible changed parameters as well as the unchanged ones as follows:

C0: the categories $1, \ldots, T$ and the utility function $u(\cdot, \cdot)$ remain unchanged.

C1: the households $M=\{1, \ldots, m\}$ change to $\hat{M}=\{\hat{1}, \ldots, \hat{m}\}$;

C2: the incomes $I_{1}, \ldots, I_{m}$ change to $\hat{I}_{\hat{1}}, \ldots, \hat{I}_{\hat{m}}$;

C3: the cost functions $C_{k}$ change to $\hat{C}_{k}(k=1, \ldots, T) ;{ }^{13}$

C4: the marginal category $f$ changes to $\hat{f}$;

C5: the boundary income $I_{G(k)}$ changes to $\hat{I}_{\hat{G}(k)}$.

The unchanged parameters are only listed in $\mathrm{C} 0$, but the other parameters listed in C1-C5 are possibly changed.

Among those parameters, the change of the $k$-th boundary income $I_{G(k)}$ to $\hat{I}_{\hat{G}(k)}$ plays an important role in our comparative study. Especially, the income difference $\hat{I}_{\hat{G}(k)}-I_{G(k)}$ is crucial. Note that this change may be caused by the other parameter changes listed in C1-C4. Kaneko et al. (2006) focused the difference $\hat{I}_{\hat{G}(k)}-I_{G(k)}$ and provided the following proposition.

Proposition 3 (Kaneko et al. (2006)). Let $r=\left(r_{1}, \ldots, r_{f}\right)$ and $\hat{r}=\left(\hat{r}_{1}, \ldots, \hat{r}_{\hat{f}}\right)$ be the differential rent vectors in $(M, N)$ and $(\hat{M}, \hat{N})$. Let $k$ be a category num-

\footnotetext{
$\overline{{ }^{13} \text { Since }}$ we have assumed that the landlords are identified with the categories, $1, \ldots, T$, the landlords remain the same, but their cost functions may change.
} 
ber with $1 \leq k \leq \min (f, \hat{f})-1$. Then,

$$
\hat{r}_{k}-r_{k} \leq \hat{r}_{k+1}-r_{k+1} \text { if and only if } \hat{I}_{\hat{G}(k)}-I_{G(k)} \leq \hat{r}_{k}-r_{k} \text {. }
$$

Note that $\leq$ in $(8)$ can be replaced by $\geq,>,<$ or $=$. Proposition 3 claims that the comparison of the rent differences at $k$ and $k+1$ is reduced into that of the boundary income difference at $k$ and the rent difference at $k .{ }^{14}$ This is the theorem to be used for our comparative statics results.

\subsection{Effects of quality changes on rents}

Now, we are ready to present the two main theorems of the present paper.

Suppose that $(M, N)$ changes into $(\hat{M}, \hat{N})$ with C0-C5 as described in Section 4.1. Let $r=\left(r_{1}, \ldots, r_{f}\right)$ and $\hat{r}=\left(\hat{r}_{1}, \ldots, \hat{r}_{\hat{f}}\right)$ be the differential rent vectors in $(M, N)$ and $(\hat{M}, \hat{N})$. Now, for the first theorem, we consider the following specific change from $(M, N)$ to $(\hat{M}, \hat{N})$. Let $k(\leq f-1)$ be a category:

$\mathrm{CS} 1: \hat{f}=f$ and $\hat{r}_{\hat{f}}=r_{f}$;

CS2: $\hat{I}_{\hat{G}(t)}=I_{G(t)}$ for all $t \leq f-1$;

CS3: $\hat{h}_{k}>h_{k}$ and $\hat{h}_{t}=h_{t}$ for all $t \leq f-1$ with $t \neq k$.

Condition CS1 states that the marginal category $f$ remains unchanged and also its rent. Condition CS2 states that all the boundary incomes remain unchanged. Condition CS3 states that a quality improvement of apartments occurs only in the $k$-th category. Thus, the parameters, other than the quality of apartments in category $k$, are fixed. It should be noted that, although $h_{k}$ increases to $\hat{h}_{k}$, it is still assumed that $\hat{h}_{k-1}>\hat{h}_{k}$. In Section 7, we will mention $\overline{{ }^{14} \text { Braid }}$ (1981) gave a differential equation similar to (8). See his equation (6). 
a case in which this assumption is eliminated.

Under the above conditions, we have the following comparative statics result, which will be proved in Section 6 .

Theorem 1. Under CS1, CS2 and CS3, we have the following:

(i) $\hat{r}_{t}=r_{t}$ for all $t=k+1, \ldots, f-1$;

(ii) $\hat{r}_{k}>r_{k}$;

(iii) $\hat{r}_{t} \leq r_{t}$ for all $t=1, \ldots, k-1$;

(iv) $0 \geq \hat{r}_{t}-r_{t} \geq \hat{r}_{t+1}-r_{t+1}$ for all $t=1, \ldots, k-2$.

When $I_{G(k-1)}>I_{G(k)}$, (iii) and (iv) hold with strict inequalities.

Fig. 3 is a schematic representation of Theorem 1. The horizontal axis expresses the ranking of categories and the vertical axis expresses apartment rents. The solid line and the dotted line depict the differential rent vectors in $(M, N)$ and $(\hat{M}, \hat{N})$, respectively. In Fig. 3, the assertions of Theorem 1 are: (i) for categories worse than $k$, the rents form one line; (ii) for category $k$, the point on the solid line jumps to that on the dotted line; but (iii) for categories better than $k$, the solid line moves down to the dotted line. These two opposite effects, (ii) and (iii), make the solid and dotted lines intersect. We will comment on assertion (iv) after presenting the next theorem. An emphasis of Theorem 1 is the contrast of the increase in $r_{k}$ and the decreases in $r_{k-1}, \ldots, r_{1}$

The above result may look counter-intuitive in the sense that we may expect increases in rents for categories better than $k$. We will argue, however, that closer examination of the economic structure will yield a natural explanation: it is, in fact, not counter-intuitive. Before the explanation, however, we present 
the second theorem, Theorem 2. This is a variant of a result in Kaneko et al. (2006), which looks opposite to the first theorem. Theorem 2 will also facilitate understanding of the first theorem.

To state Theorem 2, we keep condition CS1 but replace conditions CS2 and CS3 by

$\mathrm{CS} 2^{*}: \hat{I}_{\hat{G}(k)}>I_{G(k)}$ and $\hat{I}_{\hat{G}(t)}=I_{G(t)}$ for all $t \leq f-1$ with $t \neq k$

$\mathrm{CS}^{*}: \hat{h}_{t}=h_{t}$ for all $t \leq f-1$.

Only the boundary income of category $k$ increases, but there are no changes in the other boundary incomes - - CS2*, and all the qualities of apartments remain unchanged - - CS3*. With the above-stated replacements, we have a result opposite to Theorem 1 . A sketch of a proof will be given for completeness in Section 6.

Theorem 2. Under CS1, CS2* and $\mathrm{CS}^{*}$, we have the following:

(i) $\hat{r}_{t}=r_{t}$ for all $t=k+1, \ldots, f-1$;

(ii) $\hat{r}_{k}>r_{k}$;

(iii) $\hat{r}_{t}>r_{t}$ for all $t=1, \ldots, k-1$;

(iv) $0<\hat{r}_{t}-r_{t}<\hat{r}_{t+1}-r_{t+1}$ for all $t=1, \ldots, k-2$.

The comparative statics conditions of Theorem 2 differ from those of Theorem 1 in that only the $k$-th boundary income increases in Theorem 2 , while only the $k$-th apartment quality is improved in Theorem 1 . Their resulting effects are opposite, which are written in their third assertions. Thus, Fig. 4, depicting Theorem 2, differs from Fig. 3 in that the dotted line moves up without intersecting the solid line.

In addition to those opposite changes in rents, each theorem has the fourth 
assertion (iv) about the decrements (or increments) of rents for categories $1, \ldots, k-1$. That is, the differences determined by the solid and dotted lines are diminishing in the direction from category $k-1$ to better ones. Behavior of such decrements (or increments) was discussed in Kaneko et al. (2006), Sections 5 and 6 .

\subsection{Comparison of Theorems 1 and 2}

Now, we consider the reason why and how we have the opposite effects in Theorems 1 and 2 . Recall that in the rent equation, the $k$-th boundary household $G(k)$ is indifferent between the actual choice $\left(\boldsymbol{e}^{k}, I_{G(k)}-r_{k}\right)$ and the reference point $\left(\boldsymbol{e}^{k+1}, I_{G(k)}-r_{k+1}\right)$. With this point in mind, we examine the difference between Theorems 1 and 2. The examination will proceed in three steps: the rent changes for categories $k+1, \ldots, f$, that for $k$, and those for $1, \ldots, k-1$.

The key to the two different results of Theorems 1 and 2 is an opposite move of the utility level of the boundary household $G(k-1)$. For Theorem 1, the quality improvement of the $k$-th apartment increases the utility level of household $G(k-1)$, while for Theorem 2 , the income increase of the $k$-th boundary household $G(k)$ decreases the utility level of household $G(k-1)$. Before this key step, which is explained in the following Step 3, we begin by explaining the other two steps.

Step 1: Both Theorem 1 and Theorem 2 have no parameter changes for categories $k+1, \ldots, f$. Thus, the rents for these categories remain unchanged. This step is applied to the solid lines for $k+1, \ldots, f$ in Fig. 3 and Fig. 4.

Step 2: Consider the $k$-th boundary household in the case of Theorem 1 . In $(M, N)$, the boundary household is indifferent between the actual choice 
$\left(\boldsymbol{e}^{k}, I_{G(k)}-r_{k}\right)$ and the reference point $\left(\boldsymbol{e}^{k+1}, I_{G(k)}-r_{k+1}\right) . \operatorname{In}(\hat{M}, \hat{N})$, the $k$-th boundary household is still indifferent between the actual choice and the reference point, but no change occurs in the reference point, since $\hat{r}_{k+1}=r_{k+1}$. Thus, the $k$-th boundary household's actual utility level remains unchanged after the quality improvement $\left(\hat{h}_{k}>h_{k}\right)$, which implies a decrease in its consumption, i.e., $\hat{r}_{k}>r_{k}$.

In the case of Theorem 2, almost the same logic works with the change from the quality improvement to the income increase, in which case the normality property of the utility function is needed.

These effects are illustrated by the rent increases at $k$ in Fig. 3 and Fig. 4.

Step 3: Consider the $(k-1)$-th boundary household in the case of Theorem 1 . Note that the utility level of the $k$-th boundary household remains unchanged even after the quality improvement, and that the $(k-1)$-th boundary household has a higher income than the $k$-th boundary household. ${ }^{15}$ From the normality property of the utility function, it follows that the $(k-1)$-th boundary household prefers $\left(\hat{\boldsymbol{e}}^{k}, I_{G(k-1)}-\hat{r}_{k}\right)$ in $(\hat{M}, \hat{N})$ to $\left(\boldsymbol{e}^{k}, I_{G(k-1)}-r_{k}\right)$ in $(M, N)$, where $\hat{\boldsymbol{e}}^{k}$ denotes the improved apartment. That is, the reference point in $(\hat{M}, \hat{N})$ gives a higher utility level to the $(k-1)$-th boundary household than the corresponding reference point in $(M, N)$, which corresponds to the key mentioned above.

The quality of the $(k-1)$-th apartment does not change, and the $(k-1)$-th boundary household is indifferent between the actual choice $\left(\boldsymbol{e}^{k-1}, I_{G(k-1)}-\right.$ $\left.\hat{r}_{k-1}\right)$ and the reference point $\left(\hat{\boldsymbol{e}}^{k}, I_{G(k-1)}-\hat{r}_{k}\right)$ in $(\hat{M}, \hat{N})$. Thus, the utility premium from $\left(\boldsymbol{e}^{k-1}, I_{G(k-1)}-r_{k-1}\right)$ to $\left(\boldsymbol{e}^{k-1}, I_{G(k-1)}-\hat{r}_{k-1}\right)$ is reflected in an

\footnotetext{
$\overline{{ }^{15} \text { Here, }}$ we assume that $I_{G(k-1)}>I_{G(k)}$.
} 
increase in consumption, i.e., $I_{G(k-1)}-\hat{r}_{k-1}>I_{G(k-1)}-r_{k-1}$. Thus, $\hat{r}_{k-1}<r_{k-1}$. This is illustrated by the rent decrease at $k-1$ in Fig. 3.

Now, consider the $(k-1)$-th boundary household in the case of Theorem 2 . Here, the change in the reference point $\left(\boldsymbol{e}^{k}, I_{G(k-1)}-\hat{r}_{k}\right)$ to the $(k-1)$-th boundary household is only the increased rent $\hat{r}_{k}$, i.e., the lower consumption $I_{G(k-1)}-\hat{r}_{k}$. This implies that the utility level of the $(k-1)$-th boundary household decreases, which corresponds to the key mentioned above. Since the $(k-1)$-th boundary household's income remains unchanged, the indifference relation between $\left(\boldsymbol{e}^{k-1}, I_{G(k-1)}-\hat{r}_{k-1}\right)$ and $\left(\boldsymbol{e}^{k}, I_{G(k-1)}-\hat{r}_{k}\right)$ implies a decrease in consumption, i.e., $I_{G(k-1)}-\hat{r}_{k-1}<I_{G(k-1)}-r_{k-1}$. Thus, $\hat{r}_{k-1}>r_{k-1}$. This is illustrated by the rent increase at $k-1$ in Fig. 4 .

The argument immediately above can be extended to have $\hat{r}_{t}<r_{t}(t=$ $1, \ldots, k-2)$ in Theorem 1 and $\hat{r}_{t}>r_{t}(t=1, \ldots, k-2)$ in Theorem 2.

We have completed our explanations of Theorems 1 and 2.

Finally, we should mention certain results by Braid (1981). ${ }^{16}$ He studied the comparative statics in a housing market model with a continuous income distribution and a continuous quality distribution. He obtained results similar to Theorems 1 and 2, which are discussed in his Sections 3 and 4. His second result, when the income distribution changes only at a certain income level, is essentially the same as Theorem 2 . We should mention the difference of our Theorem 1 from his first result.

\footnotetext{
$\overline{{ }^{16} \text { Sweeney }}$ (1974) - see particularly p.164 - and van Lierop (1982) are also related to our approach. Sweeney (1974) treats housing qualities as discrete variables, while van Lierop (1982) takes them as continuous variables.
} 
The first result is similar to Theorem 1 in that when the quality distribution changes at a certain quality level, all the affected rents decrease, while in Theorem 1, all the rents other than that with a quality change decrease. ${ }^{17}$ Since housing quality is expressed by a continuous function in Braid (1981), a quality change should be made without breaking continuity. This is why his result differed from ours.

\section{Application: multiple improvements}

In Theorem 1, we assumed that an apartment quality is improved only in one category. In this section, we show that Theorem 1 can be extended to a case with improvements in multiple categories. We obtain this extension by repeated applications of Theorem 1, which is shown using the example in Section 2.

Recall the market example $(M, N)$ of Table 1 with $U(s, t, c)=s-t+\sqrt{c}$. Now, consider the change: the commuting time $t_{3}$ is shortened to $\hat{t}_{3}$. Then, utility levels $h_{3}, h_{7}$ and $h_{11}$ increase to $\hat{h}_{3}, \hat{h}_{7}$ and $\hat{h}_{11}$, respectively. Table 2 expresses this situation, where the improved categories are indicated by shading.

The above entire change is divided into the three steps indicated in Fig. 5. The first vector, $\left(h_{1}, \ldots, h_{12}\right)$, is transformed into the second vector with the change of $h_{11}$ to $\hat{h}_{11}$, and this second one is then transformed into the third one with an additional change of $h_{7}$ to $\hat{h}_{7}$. Finally, the fourth vector is obtained from the third one with the change of $h_{3}$ to $\hat{h}_{3}$. Theorem 1 is applied to each step.

$\overline{{ }^{17} \text { Note, }}$ however, that (i), (iii) and (iv) of Theorem 1 are similar to Braid's results. See FIG. 3 of Braid (1981). 
In the first application, $r_{1}, \ldots, r_{10}$ are decreased into $\hat{r}_{1}, \ldots, \hat{r}_{10}$. In the second application, $\hat{r}_{1}, \ldots, \hat{r}_{6}$ are decreased. Finally, in the third application, we have new rents for categories 1 and 2, which are respectively smaller than $r_{1}$ and $r_{2}$. As a result, we may conclude that the rents decrease for categories $1,2,4$, 6, 8 and 10, which are closer to the CBD than $t_{3} \cdot{ }^{18}$

However, when the commuting times $t_{2}$ and $t_{3}$ in Table 1 are shortened simultaneously, the above procedure cannot be directly applied. This change is expressed in Table 3. Here, apartments in consecutive categories, e.g., the 10-th and the 11-th, are improved together. In this case, we cannot apply Theorem 1. In fact, we have succeeded in generalizing Theorem 1 to treat this case, which will be done in a separate forthcoming paper.

\section{Proofs of Theorem 1 and 2}

Proof of Theorem 1. The categories in $(M, N)$ are the same in $(\hat{M}, \hat{N})$ by $\mathrm{C} 0$, and the number of equations in the rent equation (7) is the same for $(\hat{M}, \hat{N})$ by CS1. Thus, the rent equations for $(M, N)$ and $(\hat{M}, \hat{N})$ are expressed, respectively, as:

$$
\begin{aligned}
& h_{t}+g\left(I_{G(t)}-r_{t}\right)=h_{t+1}+g\left(I_{G(t)}-r_{t+1}\right) \text { for } t=1, \ldots, f-1 ; \\
& \hat{h}_{t}+g\left(\hat{I}_{\hat{G}(t)}-\hat{r}_{t}\right)=\hat{h}_{t+1}+g\left(\hat{I}_{\hat{G}(t)}-\hat{r}_{t+1}\right) \text { for } t=1, \ldots, f-1 .
\end{aligned}
$$

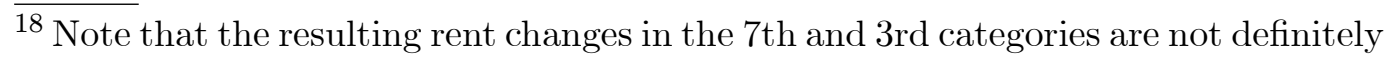
determined. Although $r_{7}$ and $r_{3}$ decrease in the first step, the 7 th rent increases in the second step, and the 3rd rent increases in the third.
} 
By CS3, $\hat{h}_{t}=h_{t}$ for all $t \leq f-1$ with $t \neq k$, and by CS2, $\hat{I}_{\hat{G}(t)}=I_{G(t)}$ for all $t \leq f-1$. Now, by subtracting (9) from (10), we have

$$
g\left(I_{G(t)}-\hat{r}_{t}\right)-g\left(I_{G(t)}-r_{t}\right)=g\left(I_{G(t)}-\hat{r}_{t+1}\right)-g\left(I_{G(t)}-r_{t+1}\right)
$$

for all $t \leq f-1$ with $t \neq k$.

(i): Consider (11) with $t=f-1$. Since $\hat{r}_{f}=r_{f}$ by CS1, we have

$g\left(I_{G(f-1)}-\hat{r}_{f-1}\right)-g\left(I_{G(f-1)}-r_{f-1}\right)=g\left(I_{G(f-1)}-\hat{r}_{f}\right)-g\left(I_{G(f-1)}-r_{f}\right)=0$.

This and the strict monotonicity of $g$ imply $I_{G(f-1)}-\hat{r}_{f-1}=I_{G(f-1)}-r_{f-1}$, that is, $\hat{r}_{f-1}=r_{f-1}$. We can repeat the above argument for $t=f-2, \ldots, k+1$, and we obtain $\hat{r}_{t}=r_{t}$ for all $t=k+1, \ldots, f-1 .{ }^{19}$

(ii): Consider the right-hand sides of (9) and (10) with $t=k$. Since $\hat{h}_{k+1}=$ $h_{k+1}$ by CS3, $\hat{I}_{\hat{G}(k)}=I_{G(k)}$ by CS2 and $\hat{r}_{k+1}=r_{k+1}$ by (i), we have:

$$
\hat{h}_{k}+g\left(I_{G(k)}-\hat{r}_{k}\right)=h_{k}+g\left(I_{G(k)}-r_{k}\right)
$$

Since $\hat{h}_{k}>h_{k}$ by CS3, we have $g\left(I_{G(k)}-\hat{r}_{k}\right)<g\left(I_{G(k)}-r_{k}\right)$. This and the strict monotonicity of $g$ imply $I_{G(k)}-\hat{r}_{k}<I_{G(k)}-r_{k}$, that is, $\hat{r}_{k}>r_{k}$.

(iii): We first show that $\hat{r}_{k-1} \leq r_{k-1}$. Let $\delta=I_{G(k-1)}-I_{G(k)} \geq 0$. Since $g$ is strictly concave, we have, from (12), $\hat{h}_{k}+g\left(I_{G(k)}-\hat{r}_{k}+\delta\right) \geq h_{k}+$ $g\left(I_{G(k)}-r_{k}+\delta\right)$, that is,

$$
\hat{h}_{k}+g\left(I_{G(k-1)}-\hat{r}_{k}\right) \geq h_{k}+g\left(I_{G(k-1)}-r_{k}\right)
$$

Using the rent equation (10) with $t=k-1$ in $(\hat{M}, \hat{N})$ and $(9)$ with $t=k-1$ in $(M, N)$, we obtain the following equation from (13):

$$
\hat{h}_{k-1}+g\left(I_{G(k-1)}-\hat{r}_{k-1}\right) \geq h_{k-1}+g\left(I_{G(k-1)}-r_{k-1}\right) .
$$

$\overline{19}$ To be exact, the induction principle must be used. 
Since $\hat{h}_{k-1}=h_{k-1}$ by CS3, we have $g\left(I_{G(k-1)}-\hat{r}_{k-1}\right) \geq g\left(I_{G(k-1)}-r_{k-1}\right)$. This and the strict monotonicity of $g$ imply $I_{G(k-1)}-\hat{r}_{k-1} \geq I_{G(k-1)}-r_{k-1}$, that is, $\hat{r}_{k-1} \leq r_{k-1}$.

Now, using (11) and $\hat{r}_{k-1} \leq r_{k-1}$, we have $g\left(I_{G(k-2)}-\hat{r}_{k-2}\right)-g\left(I_{G(k-2)}-r_{k-2}\right)$ $=g\left(I_{G(k-2)}-\hat{r}_{k-1}\right)-g\left(I_{G(k-2)}-r_{k-1}\right) \geq 0$, where the strict monotonicity of $g$ is used in the last inequality. Thus, we have $\hat{r}_{k-2} \leq r_{k-2}$. We can repeat this argument, and we have $\hat{r}_{t} \leq r_{t}$ for all $t=1, \ldots, k-1$.

(iv): By CS2 and (iii), we have $\hat{I}_{\hat{G}(1)}-I_{G(1)}=0 \geq \hat{r}_{1}-r_{1}$. Thus, by Proposition 3 (Kaneko et al. (2006)), we have $0 \geq \hat{r}_{1}-r_{1} \geq \hat{r}_{2}-r_{2}$. From this and CS2, we have $\hat{I}_{\hat{G}(2)}-I_{G(2)}=0 \geq \hat{r}_{2}-r_{2}$. By applying Proposition 3 again, we have $0 \geq \hat{r}_{2}-r_{2} \geq \hat{r}_{3}-r_{3}$. Repeating this argument, we have $0 \geq \hat{r}_{t}-r_{t} \geq \hat{r}_{t+1}-r_{t+1}$ for all $t=1, \ldots, k-2$.

When the difference $\delta=I_{G(k-1)}-I_{G(k)}$ defined in (iii) is strictly positive, the subsequent inequalities all become strict.

Sketch of the proof of Theorem 2. This theorem can be proved in an almost similar manner to the above proof of Theorem 1. The main difference is that the normality property of the utility function is used for (ii), while it was used for (iii) in the proof of Theorem 1. Another difference is that only the boundary income $I_{G(k)}$ increases in this theorem, while only $h_{k}$ increases in Theorem 1. This generates the opposite behavior stated in (iii). The other parts of the proof are essentially the same. 


\section{Conclusions}

This paper investigated the relationship between the qualities of apartments and their rents. The main result states that when a quality is improved in one category of apartments, the apartment rent for the category increases but the rents for the better categories decrease (Theorem 1). For the purpose of comparison, we gave another comparative statics result where an income increase occurs in one category (Theorem 2). In this case, the difference is that the rents increase for the better categories. Thus, we have shown a quite opposite behavior in rents with certain changes in parameters, whereas we might expect similar resulting effects. Also, we discussed the difference between our main result and Braid's (1981).

As an extension of the main result, we considered a case where qualitative improvements occur in multiple categories. The extension was considered in a rental housing market where categories are determined by commuting times and apartment sizes. As a result, we obtain the implication that when some improvement occurs in one residential area, the rents decrease in areas closer to the CBD.

Finally, we mention one possible area for future research. For Theorem 1, we assumed that the apartment ranking remains unchanged after a quality improvement. This assumption excludes some important cases. For example, suppose that a quality improvement occurs in the $k$-th category in $(M, N)$ but changes the category ranking, such as the $(k-1)$-th, in the new market $(\hat{M}, \hat{N})$. Here, we conjecture that a conclusion similar to Theorem 1 would

hold. That is, $\hat{r}_{t}<r_{t}$ for all $t=1, \ldots, k-2$ would hold for the above case. 
Although we have not succeeded in proving this conjecture, we have obtained many numerical simulation results in which the conjecture holds. The above problem and simulation results will be discussed in a separate paper.

\section{Acknowledgments}

I am grateful to Mamoru Kaneko, Richard Arnott (editor) and three anonymous referees for their incisive comments and suggestions.

\section{References}

Allonso, W., 1964. Location and Land Use. Harvard University Press, Cambridge, MA.

Arnott, R. J., 1987. Economic theory and housing. In: Mills, E. S. (Ed.), Handbook of Regional and Urban Economics. Vol. 2. North-Holland, Amsterdam, Ch. 24, pp. 959-988.

Braid, R. M., 1981. The short-run comparative statics of a rental housing market. Journal of Urban Economics 10, 286-310.

Braid, R. M., 1984. The effects of government housing policies in a vintage filtering model. Journal of Urban Economics 16, 272-296.

Fujita, M., 1989. Urban Economic Theory: Land Use and City Size. Cambridge University Press, New York.

Gerber, R. I., 1985. Existence and description of housing market equilibrium. Regional Science and Urban Economics, 383-401.

Kaneko, M., 1982. The central assignment game and the assignment markets. Journal of Mathematical Economics 11, 205-232. 
Kaneko, M., 1983. Housing market with indivisibilities. Journal of Urban Economics $13,22-50$.

Kaneko, M., Ito, T., Osawa, Y., 2006. Duality in comparative statics in rental housing markets with indivisibilities. Journal of Urban Economics 59, 142170.

Kaneko, M., Yamamoto, Y., 1986. The existence and computation of competitive equilibria in markets with an indivisible commodity. Journal of Economic Theory 38, 118-136.

Mills, E. S., 1972. Studies in the Structure of the Urban Economy. Johns Hopkins University Press, Baltimore.

Muth, R. F., 1969. Cities and Housing. University of Chicago Press, Chicago.

Ricardo, D., 1965. The Principles of Political Economy and Taxation. J. M. Dent and Sons, London, (1817: original).

Shapley, L. S., Shubik, M. J., 1972. Assignment game I: the core. International Journal of Game Theory 1, 111-130.

Sweeney, J. L., 1974. Quality, commodity hierarchies, and housing markets. Econometrica 42, 147-168.

Van Lierop, J., 1982. House price structure and market equilibrium. Journal of Urban Economics 11, 272-289.

von Böhm-Bawerk, E., 1921. Positive Theory of Capital. Books for Libraries Press, New York, (1891: original), translated by W. Smart.

von Neumann, J., Morgenstern, O., 1944. Theory of Games and Economic Behavior. Princeton University Press, Princeton. 


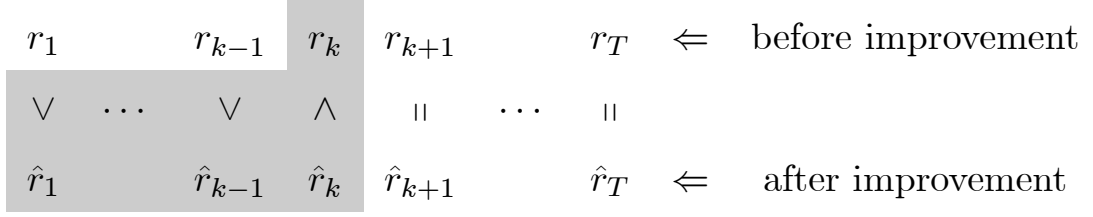

Fig. 1.

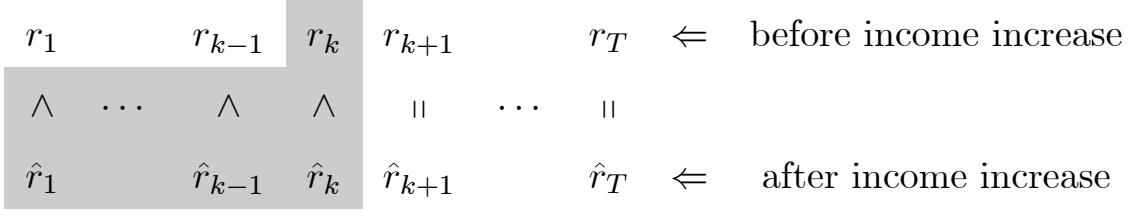

Fig. 2.

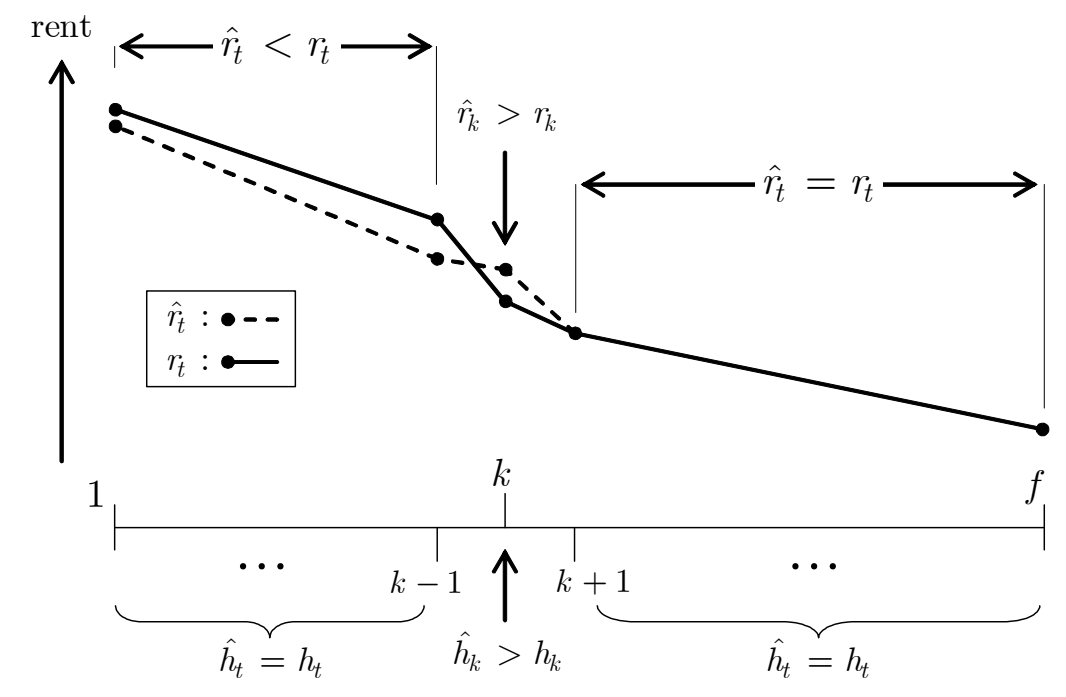

Fig. 3. Quality is improved only in the $k$-th category. 


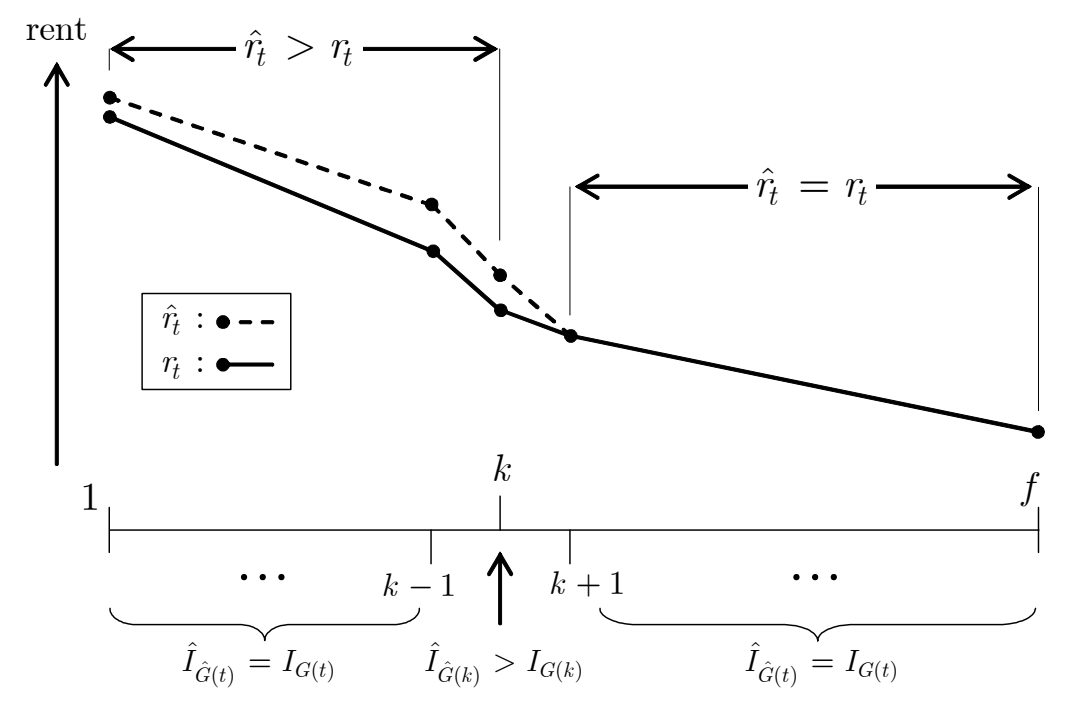

Fig. 4. Boundary income increases only in the $k$-th category.

$$
\begin{gathered}
\left(h_{1}, \ldots, h_{12}\right) \stackrel{1}{\rightarrow}\left(h_{1}, \ldots, h_{10}, \hat{h}_{11}, h_{12}\right) \stackrel{2}{\rightarrow}\left(h_{1}, \ldots, h_{6}, \hat{h}_{7}, h_{8}, h_{9}, h_{10}, \hat{h}_{11}, h_{12}\right) \\
\stackrel{3}{\rightarrow}\left(h_{1}, h_{2}, \hat{h}_{3}, h_{4}, h_{5}, h_{6}, \hat{h}_{7}, h_{8}, h_{9}, h_{10}, \hat{h}_{11}, h_{12}\right)
\end{gathered}
$$

Fig. 5. Multiple improvements. 
Table 1

Categories of apartments.

\begin{tabular}{|c|c|c|c|c|c|c|c|c|}
\hline time \size & \multicolumn{2}{|c|}{$s_{1}$} & \multicolumn{2}{|c|}{$s_{2}$} & \multicolumn{2}{|c|}{$s_{3}$} & & \\
\hline$t_{1}$ & 8 & $h_{8}$ & 4 & $h_{4}$ & 1 & $h_{1}$ & \multirow{2}{*}{\multicolumn{2}{|c|}{ legend }} \\
\hline$t_{2}$ & 10 & $h_{10}$ & 6 & $h_{6}$ & 2 & $h_{2}$ & & \\
\hline$t_{3}$ & 11 & $h_{11}$ & 7 & $h_{7}$ & 3 & $h_{3}$ & & $u_{k}$ \\
\hline$t_{4}$ & 12 & $h_{12}$ & 9 & $h_{9}$ & 5 & $h_{5}$ & & \\
\hline
\end{tabular}

Table 2

$h_{11}, h_{7}$ and $h_{3}$ increase.

\begin{tabular}{|c|c|c|c|c|c|c|c|c|}
\hline time \size & \multicolumn{2}{|r|}{$s_{1}$} & \multicolumn{2}{|r|}{$s_{2}$} & \multicolumn{2}{|r|}{$s_{3}$} & & \multirow{2}{*}{ legend } \\
\hline$t_{1}$ & 8 & $h_{8}$ & 4 & $h_{4}$ & 1 & $h_{1}$ & & \\
\hline$t_{2}$ & 10 & $h_{10}$ & 6 & $h_{6}$ & 2 & $h_{2}$ & & \\
\hline$t_{3} \rightarrow \hat{t}_{3}$ & 11 & $h_{11} \rightarrow \hat{h}_{11}$ & 7 & $h_{7} \rightarrow \hat{h}_{7}$ & 3 & $h_{3} \rightarrow \hat{h}_{3}$ & $k$ & $n_{k}$ \\
\hline$t_{4}$ & 12 & $h_{12}$ & 9 & $h_{9}$ & 5 & $h_{5}$ & & \\
\hline
\end{tabular}

Table 3

Improvements occur in consecutive categories.

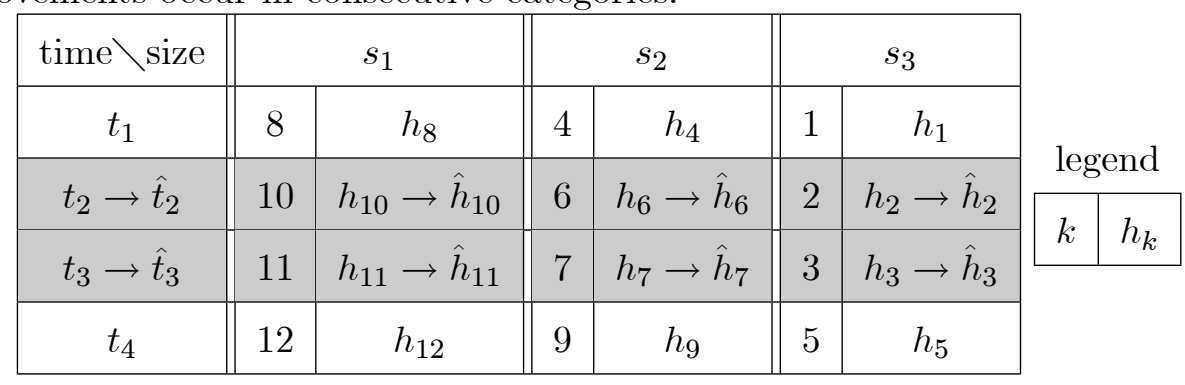

